Research Paper

\title{
Importance of maintaining body weight for prevention of distant metastasis of nasopharyngeal carcinoma: An alternative workflow for cancer-risk assessment
}

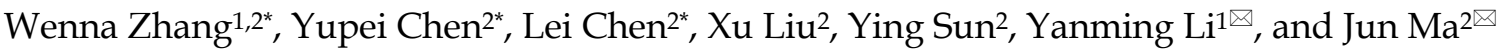 \\ 1. Department of respiratory and critical care medicine, Beijing Hospital, National Center of Gerontology, No. 1 Dahua Road, Dongdan, Beijing 100730, P.R. \\ China. \\ 2. State Key Laboratory of Oncology in South China, Department of Radiation Oncology, Sun Yat-sen University Cancer Center, Collaborative Innovation \\ Center for Cancer Medicine, Guangzhou, Guangdong, P.R. China. \\ * These authors contributed equally to this work.
}

$\square$ Corresponding author: Prof. Jun Ma, State Key Laboratory of Oncology in South China, Department of Radiation Oncology, Sun Yat-sen University Cancer Center, Collaborative Innovation Center for Cancer Medicine, 651 Dongfeng Road East, Guangzhou 510060, P.R. China. Telephone: +86-20-87343469; Fax: +86-20-87343295; E-mail: majun2@mail.sysu.edu.cn and Prof. Yanming Li, Department of respiratory and critical care medicine, Beijing Hospital, National Center of Gerontology, No. 1 Dahua Road, Dongdan, Beijing 100730, P.R. China. E-mail: liyanming2632@bjhmoh.cn

(c) Ivyspring International Publisher. This is an open access article distributed under the terms of the Creative Commons Attribution (CC BY-NC) license (https://creativecommons.org/licenses/by-nc/4.0/). See http://ivyspring.com/terms for full terms and conditions.

Received: 2017.02.11; Accepted: 2017.04.25; Published: 2017.07.20

\begin{abstract}
Purpose: To design an alternative workflow for cancer-risk assessment to predict distant metastasis (DM) of nasopharyngeal carcinoma (NPC).

Methods: We enrolled 234 patients with non-disseminated NPC and a family history of cancer who underwent intensity-modulated radiotherapy and concurrent chemo-radiotherapy with/without induction chemotherapy in our primary cohort. We conducted univariate and multivariate analyses of the associated prognostic factors, built a nomogram model for distant-metastasis-free survival (DMFS), and confirmed the prognostic value of weight-loss ratio (WTratio). The secondary cohort included 97 patients with available pre-DNA levels who were treated at our cancer center. We performed internal validation with the primary cohort and external validation with the secondary cohort, and compared the new DMFS model with the current 7th TNM staging system.

Results: In the primary cohort, $95.9 \%$ patients experienced weight loss. The $\mathrm{N}$ group (N2-3 vs. N0-1, $P=0.037)$ and pre-DNA level $(P=0.02)$ were independent prognostic factors for DMFS in NPC patients. Smoking $(P=0.051)$ and WTratio $(P=0.052)$ showed a significant trend for DMFS. WTratio was an independent prognostic factor for DMFS $(P=0.03)$. Smoking, WTratio, $\mathrm{N}$ group, and pre-DNA level were merged to build a risk-score model for DMFS using a nomogram, which could predict survival after internal and external validation.

Conclusions: Maintaining body weight during treatment is essential to prevent DM of NPC. Compared with the current 7th TNM staging system, the new DMFS model might better predict DM of NPC. The alternative workflow designed could be applied for prognostic analysis of other cancers.
\end{abstract}

Key words: nasopharyngeal carcinoma; weight-loss ratio; meta-analysis; nomogram; prognosis

\section{Introduction}

Nasopharyngeal carcinoma (NPC), also known as "Canton cancer" for its high prevalence in southern China, arises from the nasopharynx epithelium [1].
Worldwide, NPC accounted for only $0.6 \%$ of all cancers diagnosed in 2012[2]. Due to the anatomical constraints and high radiosensitivity of NPC, 


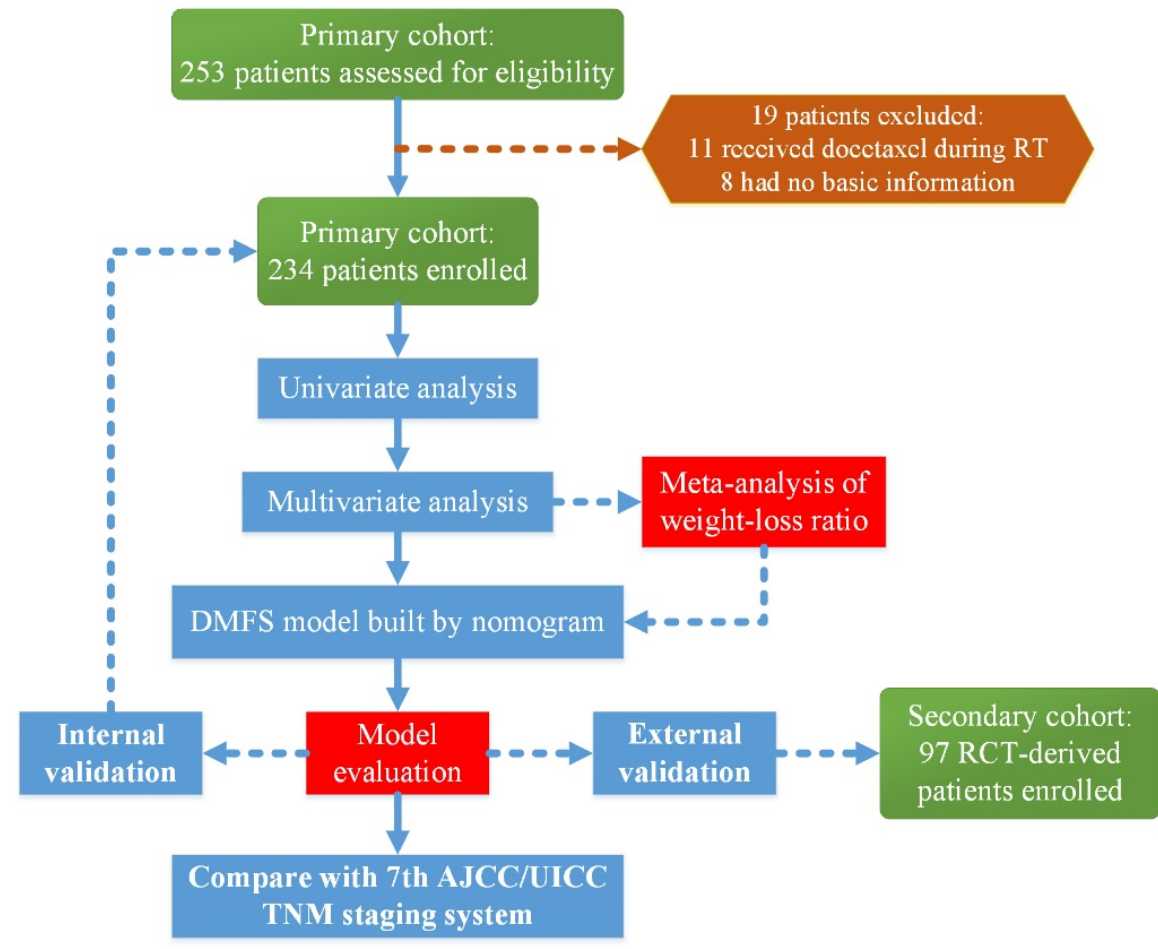

Figure 1. Flow chart of the study. Abbreviations: RT, radiotherapy; DMFS, distant-metastasis-free survival; RCT, randomized control trial; AJCC/UICC, American Joint Committee on Cancer/International Union Against Cancer

radiotherapy alone was previously the mainstay treatment, but it showed a poor overall 5-year survival rate of approximately $50 \%$ [3]. With the combination of population screening and the application of intensity-modulated radiotherapy (IMRT) and platinum-based chemotherapy, tumor control and long-term survival of NPC has improved tremendously; however, this effect was not evident for distant metastasis [2].

Our previously published meta-analysis [4] confirmed that plasma Epstein-Barr virus (EBV) DNA assays, including pre-treatment EBV DNA (pre-DNA) level, post-treatment EBV DNA (post-DNA) level, and the EBV DNA clearance rate, are highly prognostic of long-term survival and distant metastasis in patients with NPC. Other risk factors such as smoking [5], family history of cancer [6], serum lactate dehydrogenase (LDH) level [7], and lymph node staging [8] are also associated with NPC prognosis. The common side effects of treatments of cancer patients include weight loss and malnutrition, which lead to poor treatment tolerance, prolonged hospital stay, and poor outcomes [9-11]. However, despite extensive efforts to improve the outcome of patients with NPC, distant metastasis remains the main failure pattern in NPC. Therefore, this meta-analysis aimed to (1) determine the weight-loss ratio (WTratio) in a larger population and confirm the prognostic value of the WTratio, and (2) build a nomogram model for distant metastasis in order to predict the prognosis of NPC.

\section{Materials and Methods}

\subsection{Patient selection}

This study was reviewed and approved by the Ethics Committee of Sun Yat-sen University Cancer Center (SYSUCC). A total of 253 patients with a family history of cancer who received IMRT and concurrent chemo-radiotherapy (CCRT) with or without induction chemotherapy were treated at the SYSUCC between October 2009 and February 2012. We excluded 11 patients who received docetaxel during radiotherapy and eight patients whose basic information was not available. Finally, 234 patients were enrolled in the primary cohort to build the nomogram and conduct the internal validation. The secondary cohort included 97 patients with available data on the pre-DNA levels, who were treated at the SYSUCC as a part of a multi-center randomized control trial (RCT) [12] for external validation. A flow chart of the research process is presented in Figure 1.

\subsection{Data collection}

We extracted data from the medical records of patients, including sex, age, smoking, EBV infection state, pre-treatment serum LDH level, albumin titer, weight before/after treatment, and family history of cancer at diagnosis. We restaged all patients according to the 7th edition of the American Joint Committee on Cancer/International Union Against Cancer (AJCC/UICC) staging system [13]. Patients were categorized into two groups according to their family history of cancer: an NPC group, including patients with a positive history of both NPC and other cancers, and a non-NPC group, including patients without a family history of cancer. The normal serum LDH enzyme activity was defined as 109.0-245.0 $\mathrm{IU} / \mathrm{L}$, and elevated LDH activity was defined as $>245.0 \mathrm{IU} / \mathrm{L}$. The normal serum albumin level was defined as 35.0-55.0 g/L, and patients with an albumin level $<35.0 \mathrm{~g} / \mathrm{L}$ were considered to have a low albumin level. The cutoff values of pre-DNA, weight loss, and WTratio were calculated using survival receiver-operating characteristic (ROC) curves. All patients were treated with radical radiotherapy using IMRT with or without platinumbased chemo-radiotherapy. 
Table 1. Characteristics of the study and results of the univariate analysis

\begin{tabular}{|c|c|c|c|c|c|c|}
\hline \multirow[t]{2}{*}{ Factors } & \multicolumn{3}{|c|}{ Primary cohort } & \multicolumn{3}{|c|}{ Secondary cohort } \\
\hline & No. $(\%)$ & DMFS(\%) & $P$ value & No. (\%) & DMFS(\%) & $P$ value \\
\hline Age (years) & & & 0.423 & & & 0.671 \\
\hline$\geqslant 46$ & $106(45.3)$ & 84.9 & & $39(40.2)$ & 84.6 & \\
\hline$<46$ & $128(54.7)$ & 88.3 & & $58(59.8)$ & 81 & \\
\hline Sex & & & 0.358 & & & 0.031 \\
\hline Man & $174(74.4)$ & 85.6 & & $78(80.4)$ & 78.2 & \\
\hline Woman & $60(25.6)$ & 90 & & 19(19.6) & 100 & \\
\hline Smoking & & & 0.038 & & & 0.329 \\
\hline Yes & 98 (41.9) & 81.6 & & $45(46.4)$ & 86.7 & \\
\hline No & $136(58.1)$ & 90.4 & & $52(53.6)$ & 78.8 & \\
\hline FHC type & & & 0.678 & & & \\
\hline NPC & $84(35.9)$ & 85.7 & & $16(16.5)$ & & \\
\hline non-NPC & $150(64.1)$ & 87.3 & & / & & \\
\hline $\mathrm{T}$ group & & & 0.128 & & & 0.957 \\
\hline $\mathrm{T} 1 / \mathrm{T} 2$ & $73(31.2)$ & 91.8 & & $12(12.3)$ & 83.3 & \\
\hline $\mathrm{T} 3 / \mathrm{T} 4$ & $161(68.8)$ & 84.5 & & $85(87.7)$ & 82.4 & \\
\hline $\mathbf{N}$ group & & & $<0.0001$ & & & 0.885 \\
\hline N0/N1 & $186(79.5)$ & 90.9 & & $37(38.1)$ & 81.1 & \\
\hline N2/N3 & $48(20.5)$ & 70.8 & & $60(61.9)$ & 83.3 & \\
\hline Staging & & & 0.01 & & & 0.967 \\
\hline I/II/III & $139(59.4)$ & 91.4 & & $67(69.1)$ & 82.1 & \\
\hline IV & 95 (40.6) & 80 & & $30(30.9)$ & 83.3 & \\
\hline IC/AC & IC & & 0.077 & $\mathrm{AC}$ & & 0.53 \\
\hline Yes & $123(52.6)$ & 91 & & $44(45.4)$ & 79.5 & \\
\hline No & $111(47.4)$ & 82.9 & & $53(54.6)$ & 84.9 & \\
\hline LDH (IU/L) & & & 0.763 & & & \\
\hline$\geqslant 245.0$ & $13(5.6)$ & 84.6 & & / & & \\
\hline$<245.0$ & $220(94)$ & 86.8 & & / & & \\
\hline $\operatorname{Alb}(g / l)$ & & & 0.044 & & & \\
\hline$\geqslant 35.0$ & $231(98.7)$ & 87 & & / & & \\
\hline$<35.0$ & $2(0.9)$ & 50 & & / & & \\
\hline \multicolumn{2}{|c|}{ Pre-DNA (copy/ml) } & & $<0.0001$ & & & 0.757 \\
\hline$\geqslant 39.16$ & $76(32.5)$ & 81 & & $71(73.2)$ & 81.7 & \\
\hline$<39.16$ & $158(67.5)$ & 98.7 & & $26(26.8)$ & 84.6 & \\
\hline \multicolumn{2}{|c|}{ Pre-BMI (kg/m²) } & & 0.456 & & & 0.216 \\
\hline$\geqslant 23.0$ & $125(53.4)$ & 85.6 & & $46(47.4)$ & 89.1 & \\
\hline$<23.0$ & $108(46.2)$ & 88.9 & & $48(49.5)$ & 79.2 & \\
\hline \multicolumn{2}{|c|}{ Pt-BMI (kg/m²) } & & 0.063 & & & 0.728 \\
\hline$\geqslant 23.0$ & $52(22.2)$ & 94.2 & & $16(16.5)$ & 87.5 & \\
\hline$<23.0$ & $170(72.6)$ & 84.1 & & $75(77.3)$ & 82.7 & \\
\hline \multicolumn{2}{|l|}{ WT loss (kg) } & & 0.015 & & & 0.92 \\
\hline$\geqslant 4$ & $129(55.1)$ & 82.2 & & $72(74.2)$ & 83.3 & \\
\hline$<4$ & $92(39.3)$ & 93.5 & & 19(19.6) & 84.2 & \\
\hline \multicolumn{2}{|l|}{ WT ratio (\%) } & & 0.019 & & & 0.283 \\
\hline$\geqslant 5$ & $166(70.9)$ & 83.7 & & $87(89.7)$ & 83.9 & \\
\hline$<5$ & $55(23.5)$ & 96.4 & & $10(10.3)$ & 70 & \\
\hline
\end{tabular}

Abbreviations: DMFS, distant-metastasis-free survival; FHC, family history of cancer; IC, induction chemotherapy; AC, adjuvant chemotherapy; WT loss, weigh loss; WT ratio, weight loss ratio; Pre-DNA, pre-treatment Epstein-Barr virus DNA; $\mathrm{Alb}$, albumin; LDH, serum lactate dehydrogenase; Pre-BMI, pre-treatment body mass index; Pt-BMI, post-treatment body mass index.

\subsection{Meta-analysis}

We identified all studies focusing on weight loss and prognosis of NPC patients in the PubMed, Web of Science, and the Cochrane library (last search updated in June 2016) databases. Responding letters, review articles, case reports, and experimental animal studies were excluded. Two investigators (WNZ and YPC) independently extracted data from eligible studies and finally reached a consensus for all items. Additionally, we recorded the first author, year of publication, number of patients analyzed, TNM staging, cutoff value of WTratio, treatment regimens, and inclusion period for each study.

\subsection{Construction and validation of the nomogram}

Our primary endpoint was distantmetastasis-free survival (DMFS), and our secondary endpoint was overall survival (OS). We designed a nomogram for DMFS with commonly identified prognostic factors including smoking, $\mathrm{N}$ group (N0-1 vs. N2-3), TNM staging, WTratio, weight loss, pre-DNA level, and LDH level. In addition, we constructed an internal calibration plot and an external calibration plot to determine whether the observed and predicted probabilities for survival were in concordance with bootstrap resampling (500 resamples). Finally, we compared the DMFS model with the current 7 th TNM staging using the area under the curve (AUC) of the ROC curve.

\subsection{Statistical methods}

Statistical analyses were performed using the Statistical Package for the Social Sciences version 22.0 (SPSS, Chicago, IL, USA) and the Hmisc, grid, rms, lattice, ggplot2, survival ROC package in R software, version 3.3.0 (http://www.r-project.org/). Survival curves were compared using the log-rank test and calculated using the Kaplan-Meier method. Multivariate analysis was performed to test independent significance with the Cox proportional hazards model. Results were presented as hazard ratios (HRs) and $95 \%$ confidence intervals (CIs). P-values $<0.05$ were defined as statistically significant. The meta-analysis was performed using Review Manager 5.3.5 (Cochrane Collaboration, Oxford, UK) with the fixed-effect model (Mantel-Haenszel), which assumes that differences between the results of various studies are due to chance. The $\chi^{2}$-test was used to evaluate the heterogeneity across studies, and statistically significant heterogeneity was defined as a $\chi^{2} \mathrm{P}$-value $<$ 0.1 . The nomogram was constructed based on the primary cohort with the Cox model parameter estimates by using a backward step-down-selection process. The construction and validation of the nomogram were performed under Iasonos' guidance [14]. The comparisons of the AUCs were made using the method of DeLong et al [15].

\section{Results}

\subsection{Clinical features}

The primary cohort consisted of 234 eligible patients (men, 74.4\%; women 25.6\%) with nondisseminated NPC. All patients with a positive family history of cancer received IMRT and CCRT with or 
without induction chemotherapy. Approximately $35.9 \%$ of the patients with a positive history had NPC. Further, about half $(52.6 \%)$ of the patients in the cohort received induction chemotherapy. We calculated the pre-treatment and post-treatment body mass index (BMI) and found that the percentile of patients with $\mathrm{BMI} \geq 23.0 \mathrm{~kg} / \mathrm{m}^{2}$ reduced from 53.4 before chemo-radiotherapy to 22.2 after chemoradiotherapy. In total, $95.9 \%$ of the patients experienced different extents of weight loss: $55.1 \%$ lost $>4 \mathrm{~kg}$ and $70.9 \%$ lost $>5 \%$ body weight. The second cohort was derived from a multi-center RCT [12]. For external validation of the DMFS model, we selected 97 patients with available data on pre-DNA levels, who were treated at our cancer center. The patient characteristics and results of the univariate analysis for the two cohorts are shown in Table 1.

\subsection{Univariate analysis}

In univariate analysis of the primary cohort, smoking $(P=0.038)$ and hypoalbuminemia $(P=0.044)$ were significant prognostic factors for DMFS. In addition, the N0-1 group had a higher DMFS rate than the N2-3 group (90.9\% vs. $70.8 \%, P<0.0001)$. We divided the TNM staging into TNM I-III and TNM IV groups. The 4-year DMFS rate of stage IV patients was only $80 \%$, which was $11.4 \%$ lower than of stage I-III patients. The 4-year cutoff values for pre-DNA levels, weight loss, and WTratio for DMFS were 39.16 copies $/ \mathrm{ml}(\mathrm{AUC}=0.694), 4 \mathrm{~kg}(\mathrm{AUC}=0.628)$, and $5 \%$ $(\mathrm{AUC}=0.607)$, respectively. In univariate analysis, the pre-DNA, weight loss, and WTratio were all associated with DMFS $(P<0.0001, P=0.015, P=0.019$, respectively). Furthermore, the two cohorts were similar in terms of these prognostic factors (Table 1).

\subsection{Multivariate analysis}

In multivariate analysis of DMFS based on the primary cohort, the $\mathrm{N}$ group (HR: 2.234, 95\%CI: 1.050-4.752, $P=0.037$ ) and pre-DNA level (HR: 10.816, 95\%CI: $1.447-80.850, P=0.02$ ) were independent prognostic factors for DMFS of NPC. Smoking (HR: 2.103, 95\%CI: 0.996-4.439, $P=0.051)$ and WTratio (HR: 4.566, 95\%CI: 0.986-21.153, $P=0.052$ ) had a statistically significant trend for DMFS. Moreover, hypoalbuminemia was strongly related to poor survival (HR: 17.124, 95\% CI: 1.901-154.229, $P=0.011$ ). However, only 2 patients had hypoalbuminemia, and therefore, this result should be carefully interpreted. Results of the multivariate analysis of DMFS and OS are presented in Table 2. The cutoff values of age, weight loss, WTratio, and pre-DNA levels for the 4-year OS were calculated in the same way as for 4-year DMFS by survival ROC.
Table 2. Results of the multivariate analysis of the primary cohort

\begin{tabular}{llllll}
\hline Outcome & Variate & Cutoff point & HR & $\mathbf{9 5 \%}$ CI & $\boldsymbol{P}$ value \\
\hline OS & Smoking & Yes vs. No & 2.250 & $0.984-5.144$ & 0.055 \\
& Staging & IV vs. I-III & 2.568 & $1.097-6.009$ & 0.03 \\
& Age (years) & $\geqslant 46$ vs. $<46$ & 2.468 & $1.062-5.737$ & 0.036 \\
& WT loss (kg) & $\geqslant 4.0$ vs. $<4.0$ & 1.775 & $0.503-6.256$ & 0.372 \\
& WT ratio (\%) & $\geqslant 10.0$ vs. $<10.0$ & 2.671 & $1.174-6.080$ & 0.019 \\
& Pre-DNA & $\geqslant 1640$ vs. $<1640$ & 2.002 & $0.756-5.301$ & 0.162 \\
DMFS & (copy/ml) & & & & \\
& Smoking & Yes vs. No & 2.103 & $0.996-4.439$ & 0.051 \\
& Staging & IV vs. I-III & 0.987 & $0.453-2.147$ & 0.973 \\
& N group & $2-3$ vs. $0-1$ & 2.234 & $1.050-4.752$ & 0.037 \\
& WT loss (kg) & $\geqslant 4.0$ vs. $<4.0$ & 1.221 & $0.413-3.609$ & 0.719 \\
& WT ratio (\%) & $\geqslant 5.0$ vs. $<5.0$ & 4.566 & $0.986-21.153$ & 0.052 \\
& Pre-DNA & $\geqslant 39.16$ vs. $<39.16$ & 10.816 & $1.447-80.850$ & 0.02 \\
& (copy $/ \mathrm{ml})$ & & & & \\
Alb (g/l) & $<35.0$ vs. $\geqslant 35.0$ & 17.124 & $1.901-154.229$ & 0.011 \\
\hline
\end{tabular}

Abbreviations: DMFS, distant-metastasis-free survival; OS, overall survival; WT loss, weight loss; WT ratio, weight loss ratio; pre-DNA, pretreatment Epstein-Barr virus DNA; Alb, albumin, HR, hazard ratio

\subsection{Meta-analysis of the WTratio based on published data}

\subsubsection{Eligible studies}

A total of 46 studies were retrieved using the initial search algorithm, of which 4 studies [16-19] with 8 subsets were finally included $(n=5305$ patients). We performed a meta-analysis of the WTratio based on the published data and the results of our primary cohort. All the studies focused on patients with newly diagnosed non-disseminated NPC who received radiotherapy or chemoradiotherapy. Most studies [16-18] considered 5\% as the cutoff value for WTratio, except one study [19]. Analysis of our unpublished data confirmed the cutoff values of $5 \%$ for DMFS (AUC $=0.607$ ) and $10 \%$ (AUC $=0.622$ ) for OS using R software. The analyzed survival period was from 3-5 years. Characteristics of the eligible studies or subsets are listed in Table 3.

\subsubsection{Data synthesis and publication bias}

A total of 8 studies or subsets reported negative association between the WTratio and DMFS, whereas only one study [16] reported that patients with a WTratio $>10 \%$ had a high risk of distant metastasis as compared to patients with a WTratio $<5 \%$. On pooling the data of 9 studies or subsets, we found that the WTratio was an independent prognostic factor for DMFS (HR: 1.27, 95\%CI: 1.02-1.58, $P=0.03$ ). Furthermore, there was no significant between-study heterogeneity $\left(X^{2}=10.3, I^{2}=22 \%, P=0.24\right)$. We constructed a funnel plot for DMFS, which indicated no obvious publication bias (Supplementary Fig. S1). We also performed a meta-analysis of the OS and confirmed the prognostic value of the WTratio (HR: 1.53, 95\% CI: 1.37-1.71, $P<0.00001)$ among 3 negative subsets and 6 positive subsets. A forest plot of DMFS and OS is presented in Figure 2. 


\subsection{DMFS model built by the nomogram}

A nomogram was developed to predict the 4-year DMFS based on the results of the multivariate analysis (Figure 3). Since only 2 patients had hypoalbuminemia in the primary cohort, the results for hypoalbuminemia might not be representative of the whole population. Therefore, albumin was not included in the development of the nomogram model. As shown in Figure 4, smoking, WTratio, N group, and pre-DNA levels were finally considered together to build the risk-score model for DMFS. A high pre-DNA level and high WTratio accounted for 10 points and 5 points, respectively. Moreover, the N2-3 patients gained 3 points according to the model. Smoking gained approximately 2.8 points. The total points ranged from 0 to 24 , and the corresponding prediction of 1-year to 4-year DMFS was calculated and shown below the total points.

Table 3. Characteristics of the eligible studies in the meta-analysis of weight loss ratio

\begin{tabular}{|c|c|c|c|c|c|c|c|}
\hline Studies & Subsets & Inclusion period & Reported survival (years) & Total patients & Stage & Cutoff value & Treatments \\
\hline Lin et al. & & 2006-2012 & 3 & 238 & $\mathrm{I}-\mathrm{IVb}$ & $5 \%$ & RT/CRT \\
\hline Du et al. & $5 \%-10 \%$ vs. $5 \%$ & 2003-2006 & 5 & 507 & $\mathrm{I}-\mathrm{IVb}$ & $5 \%$ & $\mathrm{RT} / \mathrm{CRT}$ \\
\hline Du et al. & $10 \%$ vs. $5 \%$ & 2003-2006 & 5 & 422 & $\mathrm{I}-\mathrm{IVb}$ & $5 \%$ & $\mathrm{RT} / \mathrm{CRT}$ \\
\hline Shen et al. & Hypo-weight & 2000-2004 & 5 & 212 & $\mathrm{I}-\mathrm{IVb}$ & $5 \%$ & $\mathrm{RT} / \mathrm{CRT}$ \\
\hline Shen et al. & Normal weight & 2000-2004 & 5 & 1157 & $\mathrm{I}-\mathrm{IVb}$ & $5 \%$ & $\mathrm{RT} / \mathrm{CRT}$ \\
\hline Shen et al. & Hyper-weight & 2000-2004 & 5 & 1064 & $\mathrm{I}-\mathrm{IVb}$ & $5 \%$ & $\mathrm{RT} / \mathrm{CRT}$ \\
\hline Zeng et al. & RT plus CCRT & 2001-2005 & 5 & 1374 & $\mathrm{I}-\mathrm{IVb}$ & $4.60 \%$ & RT/CRT \\
\hline Zeng et al. & IMRT & 2001-2005 & 5 & 110 & $\mathrm{I}-\mathrm{IVb}$ & $4.60 \%$ & $\mathrm{RT} / \mathrm{CRT}$ \\
\hline Primary cohort & & 2009-2012 & 4 & 221 & $\mathrm{I}-\mathrm{IVb}$ & $\begin{array}{l}\text { DMFS:5\% } \\
\text { OS: } 10 \%\end{array}$ & CCRT/IC+CCRT \\
\hline
\end{tabular}

Abbreviations: RT, radiotherapy; CRT, chemoradiotherapy; CCRT, concurrent chemoradiotherapy; DMFS, distant-metastasis-free survival; IC, induction chemotherapy; IMRT, intensity-modulated radiotherapy

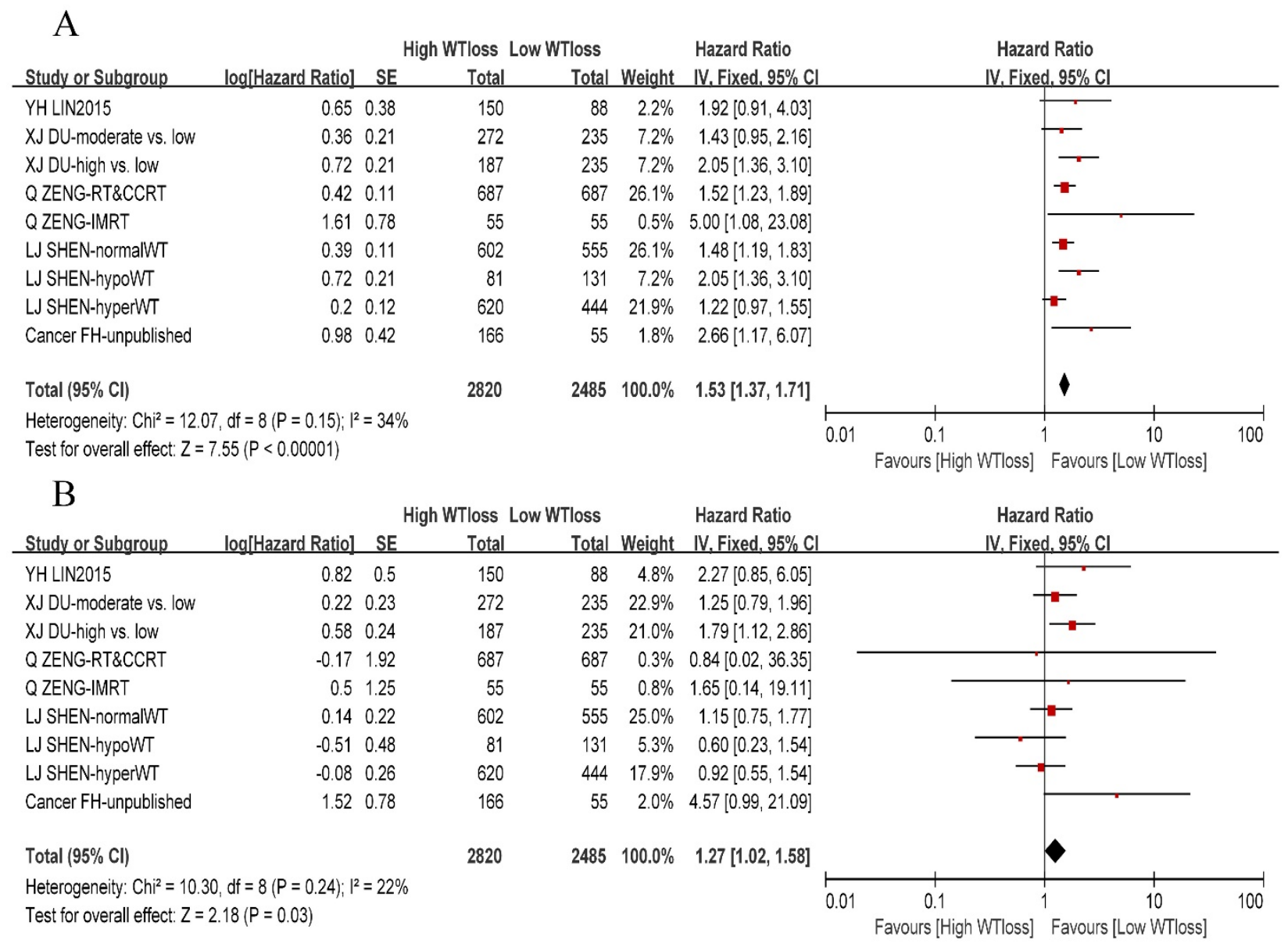

Figure 2. Forest plot of the weight-loss ratio associated with overall survival and distant-metastasis-free survival. (A) Overall survival. (B) Distant-metastasis-free survival. Abbreviations: WTloss, weight-loss ratio; Cl, confidence interval 


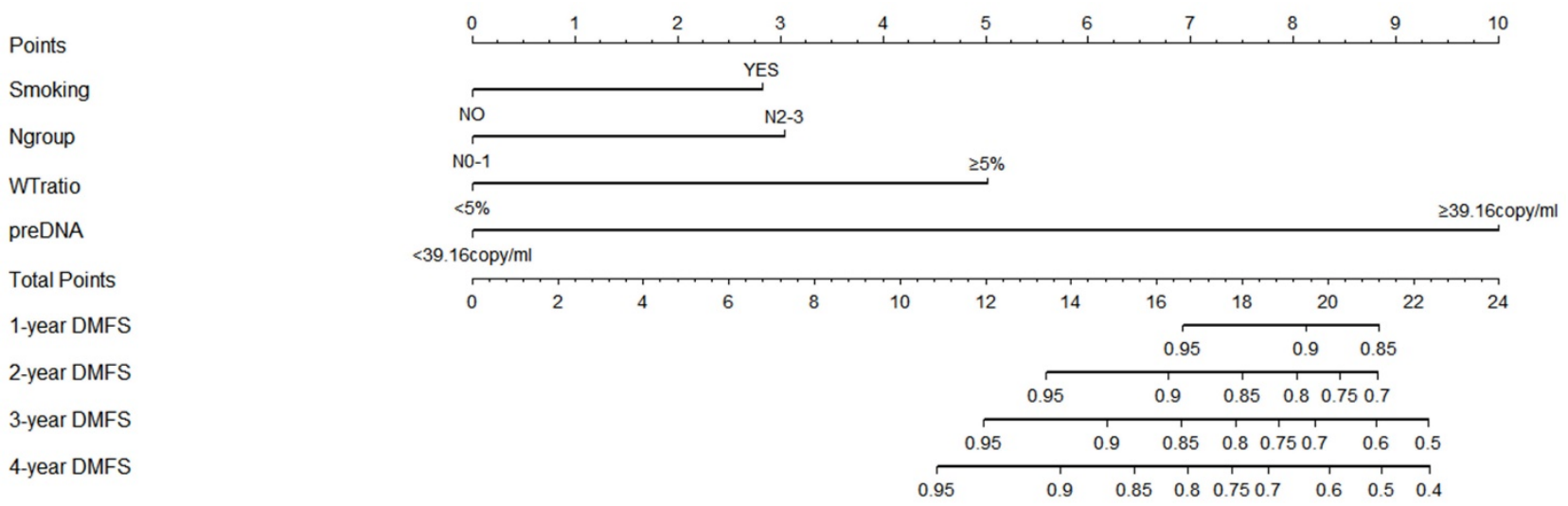

Figure 3. Nomogram for distant-metastasis-free survival of nasopharyngeal carcinoma based on the results of multivariate analysis. High pre-DNA levels and high weight-loss ratio accounted for 10 points and 5 points, respectively. N2-3 patients gained 3 points according to the model. Smoking gained approximately 2.8 points compared with the 10 points allotted to elevated pre-DNA levels. The total points ranged from 0 to 24 , and the corresponding prediction of 1 -year to 4 -year DMFS was calculated and shown below the total points. Abbreviations: preDNA, pre-DNA, pre-treatment Epstein-Barr virus DNA; DMFS, distant-metastasis-free survival; Ngroup, N group.

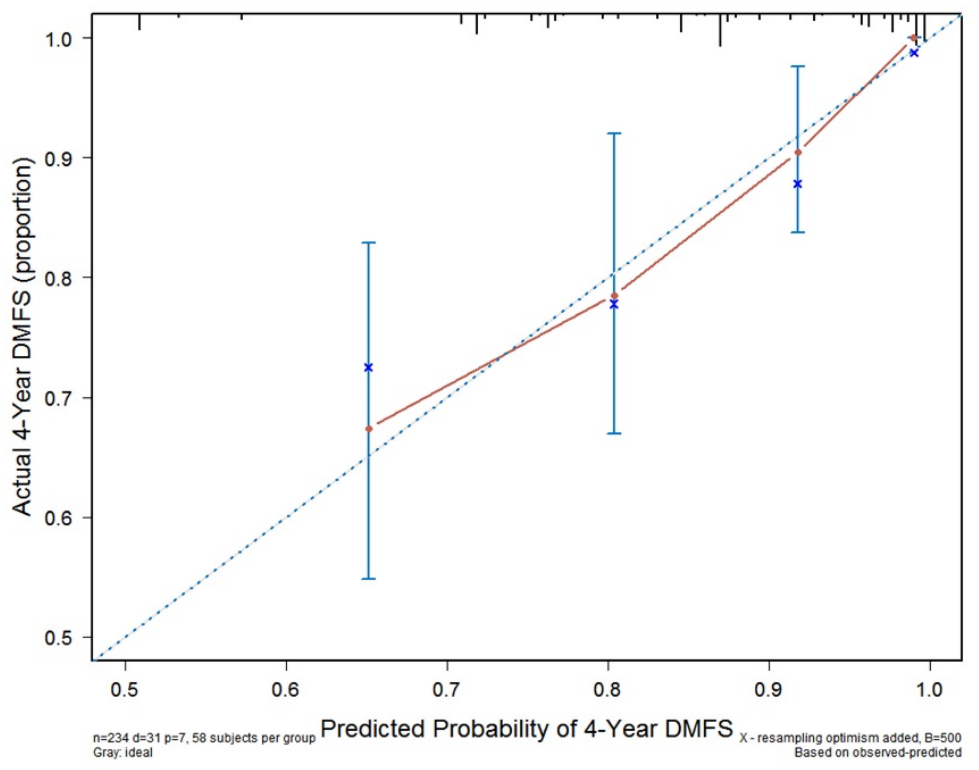

A. Internal validation based on the primary cohort.

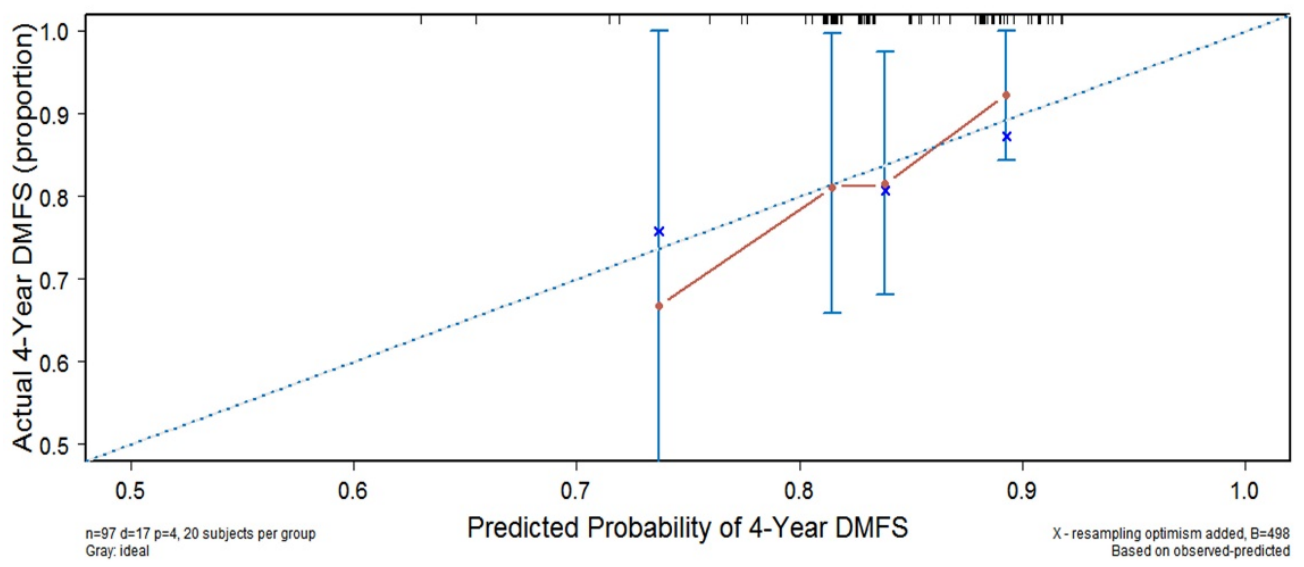

B. External validation based on the secondary cohort.

Figure 4. Internal validation and external validation of the nomogram for distant-metastasis-free survival. (A) Internal validation based on the primary cohort. (B) External validation based on the secondary cohort. Abbreviations: DMFS, distant-metastasis-free survival. 


\subsection{Evaluation of DMFS model}

\subsubsection{Internal validation}

The internal calibration plot for the 4-year DMFS performed with the primary cohort is shown in Figure 4a. The predicted 4-year DMFS showed a good correlation with the actual 4-year DMFS.

\subsubsection{External validation}

The external calibration plot for 4-year DMFS performed with the secondary cohort is shown in Figure $4 \mathrm{~b}$. The predicted 4-year DMFS showed a good correlation with the actual 4-year DMFS.

\subsubsection{Comparison with $7^{\text {th }}$ AJCC/UICC TNM staging system}

Compared with the current 7th TNM staging system $(\mathrm{AUC}=0.661,95 \% \mathrm{CI}: 0.596-0.721)$, the newly built DMFS model (AUC $=0.791,95 \% \mathrm{CI}$ : 0.733-0.841) seemed to be a better model to predict distant metastasis of NPC ( $\mathrm{Z}=2.894,95 \% \mathrm{CI}$ : $0.042-0.218, P=$ 0.0038). The comparison of the AUC curves for both systems is shown in Figure 5.

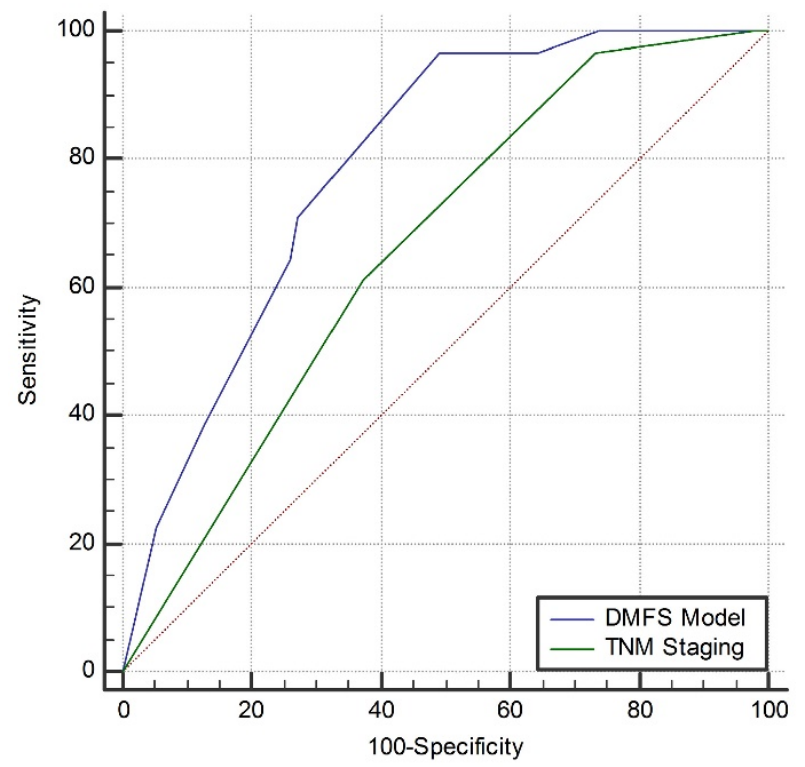

Figure 5. Comparison of the area under the curve of the receiver-operating characteristic curve between the distant-metastasis-free survival model and the current 7th TNM staging system. Abbreviations: DMFS, distant-metastasis-free survival

\section{Discussion}

In this study, we successfully developed a new DMFS model using a nomogram for predicting distant metastasis of NPC. Furthermore, we also designed an alternative workflow for cancer-risk assessment. Our study showed that weight loss was associated with the occurrence of distant metastasis. Therefore, maintaining body weight during treatment of NPC is important to prevent distant metastasis in patients with NPC.

Weight loss is a common side effect during chemotherapy or radiotherapy in patients with head and neck cancer, especially NPC; the average weight loss in patients is reported to be $5.5-12.3 \mathrm{~kg}$, and $86 \%$ of patients lose $>10 \%$ body weight [20-22]. In our primary cohort, $95.9 \%$ patients had experienced different extents of weight loss, with an average of 5.7 $\mathrm{kg}$, which was similar to that in previous studies. Stage IV NPC is a predictive marker for weight loss and low serum albumin levels [23]. In this study, stage IV patients comprised $40.6 \%$ of the primary cohort (Table 1), and most NPC patients were newly diagnosed with stage III or stage IV NPC. In our meta-analysis of 9 subsets, we confirmed that WTratio was an independent prognostic factor for DMFS, and the funnel plot for DMFS indicated no obvious publication bias. As such, nutritional management such as a prophylactic percutaneous endoscopic gastrostomy [22] might improve survival and quality of life in for patients with late-stage cancer. Combined with smoking, pre-DNA level, and $\mathrm{N}$ group, we developed a new nomogram model for DMFS, which showed a favorable predictive accuracy of 4 -year distant metastasis. The difference between the C-index for the nomogram model (AUC $=0.791)$ and the current 7th TNM staging system $(\mathrm{AUC}=0.661)$ was statistically significant $(Z=2.894,95 \% \mathrm{CI}$ : $0.042-0.218, P=0.0038$ ), which indicated a new direction for management of treatment in NPC patients.

Although the nomogram model could predict DMFS with good accuracy, it had some limitations that must be taken into account while interpreting the results of this study. First, the nomogram was developed on the basis of the multivariate analysis results of our small study sample, which might not account for all the prognostic factors associated with distant metastasis. Second, we conducted a meta-analysis to confirm the prognostic value of WTratio. However, the published data were limited to non-RCTs and mostly comprised retrospective studies. Third, we constructed the nomogram model considering patients belonging to endemic areas. It is currently unclear whether this model is applicable to patients worldwide. Although we conducted an external validation in a subgroup of patients from a published RCT, further well-designed validation trials are required to confirm our findings. One main advantage of this study was that it was performed at a time when sufficient data was available for evaluation by workflow. This workflow made use of different evidence-based statistic methods and thus could get more reliable results. And this workflow could also be 
applied to other cancers.

In conclusion, we developed and externally validated a nomogram model that can predict 4-year DMFS of NPC. This model may have better diagnostic value than the current 7th TNM staging system. It is important to maintain weight during the treatment of NPC in order to prevent distant metastasis in patients with NPC. The alternative work-flow for cancer risk assessment designed in this study could be applied to prognostic analysis of other cancers in the future.

\section{Supplementary Material}

Supplementary figure S1.

http://www.jcancer.org/v08p2269s1.pdf

\section{Acknowledgements}

We would like to thank the native English speaking scientists of Elixigen Company for editing our manuscript. We thank the anonymous reviewers for their insightful comments and great efforts to improve this manuscript.

\section{Financial Support}

This work was supported by grants from the National Science \& Technology Pillar Program during the Twelfth Five-year Plan Period (2014BAI09B10); the Health \& Medical Collaborative Innovation Project of Guangzhou City, China (201400000001); the Planned Science and Technology Project of Guangdong Province (2013B020400004); and the Science and Technology Project of Guangzhou City, China (14570006).

\section{Competing Interests}

The authors have declared no conflicts of interest.

\section{References}

1. Wei WI, Sham JS. Nasopharyngeal carcinoma. Lancet. 2005; 365: 2041-54.

2. Chua ML, Wee JT, Hui EP, Chan AT. Nasopharyngeal carcinoma. Lancet. 2016; 387: 1012-24

3. Lin JC, Chen KY, Jan JS, Hsu CY. Partially hyperfractionated accelerated radiotherapy and concurrent chemotherapy for advanced nasopharyngeal carcinoma. Int J Radiat Oncol Biol Phys. 1996; 36: 1127-36.

4. Zhang W, Chen Y, Chen L, Guo R, Zhou G, Tang L, et al. The clinical utility of plasma Epstein-Barr virus DNA assays in nasopharyngeal carcinoma: the dawn of a new era?: a systematic review and meta-analysis of 7836 cases. Medicine (Baltimore). 2015; 94: e845.

5. Chen C, Shen LJ, Li BF, Gao J, Xia YF. Smoking is a poor prognostic factor for male nasopharyngeal carcinoma treated with radiotherapy. Radiother Oncol. 2014; 110: 409-15.

6. Zhang W, Chen Y, Zhou G, Liu X, Chen L, Tang L, et al. Pretreatment Serum Lactate Dehydrogenase and N Classification Predict Long-Term Survival and Distant Metastasis in Patients With Nasopharyngeal Carcinoma Who Have A Positive Family History of Cancer. Medicine (Baltimore). 2015; 94: e1505.

7. Li G, Gao J, Tao YL, Xu BQ, Tu ZW, Liu ZG, et al. Increased pretreatment levels of serum LDH and ALP as poor prognostic factors for nasopharyngeal carcinoma. Chinese journal of cancer. 2012; 31: 197-206.

8. Sun Y, Tang LL, Chen L, Li WF, Mao YP, Liu LZ, et al. Promising treatment outcomes of intensity-modulated radiation therapy for nasopharyngeal carcinoma patients with N0 disease according to the seventh edition of the AJCC staging system. BMC Cancer. 2012; 12: 68.

9. Marin Caro MM, Laviano A, Pichard C. Nutritional intervention and quality of life in adult oncology patients. Clin Nutr. 2007; 26: 289-301.
10. Munshi A, Pandey MB, Durga T, Pandey KC, Bahadur S, Mohanti BK. Weight loss during radiotherapy for head and neck malignancies: what factors impact it? Nutr Cancer. 2003; 47: 136-40.

11. Pressoir M, Desne S, Berchery D, Rossignol G, Poiree B, Meslier M, et al. Prevalence, risk factors and clinical implications of malnutrition in French Comprehensive Cancer Centres. Br J Cancer. 2010; 102: 966-71.

12. Chen $\mathrm{L}, \mathrm{Hu} \mathrm{CS}$, Chen $\mathrm{XZ}$, Hu GQ, Cheng ZB, Sun $\mathrm{Y}$, et al. Concurrent chemoradiotherapy plus adjuvant chemotherapy versus concurrent chemoradiotherapy alone in patients with locoregionally advanced nasopharyngeal carcinoma: a phase 3 multicentre randomised controlled trial. Lancet Oncol. 2012; 13: 163-71.

13. Edge SB, Compton CC. The American Joint Committee on Cancer: the 7th edition of the AJCC cancer staging manual and the future of TNM. Annals of surgical oncology. 2010;17(6):1471-1474.

14. Iasonos A, Schrag D, Raj GV, Panageas KS. How to build and interpret a nomogram for cancer prognosis. J Clin Oncol. 2008; 26: 1364-70.

15. DeLong ER, DeLong DM, Clarke-Pearson DL. Comparing the areas under two or more correlated receiver operating characteristic curves: a nonparametric approach. Biometrics 1988; 44: 837-45.

16. Du XJ, Tang LL, Mao YP, Guo R, Sun Y, Lin AH, et al. Value of the prognostic nutritional index and weight loss in predicting metastasis and long-term mortality in nasopharyngeal carcinoma. J Transl Med. 2015; 13: 364.

17. Lin YH, Chang KP, Lin YS, Chang TS. Evaluation of effect of body mass index and weight loss on survival of patients with nasopharyngeal carcinoma treated with intensity-modulated radiation therapy. Radiat Oncol. 2015; 10: 136.

18. Shen LJ, Chen C, Li BF, Gao J, Xia YF. High weight loss during radiation treatment changes the prognosis in under-/normal weight nasopharyngeal carcinoma patients for the worse: a retrospective analysis of 2433 cases. PLoS One. 2013; 8: e68660.

19. Zeng Q, Shen LJ, Guo X, Guo XM, Qian CN, Wu PH. Critical weight loss predicts poor prognosis in nasopharyngeal carcinoma. BMC Cancer. 2016; 16: 169

20. Bahl M, Siu LL, Pond GR, Kim J, Tannock IF, Bayley A, et al. Tolerability of the Intergroup 0099 (INT 0099) regimen in locally advanced nasopharyngeal cancer with a focus on patients' nutritional status. Int J Radiat Oncol Biol Phys. 2004; 60: 1127-36.

21. Fountzilas G, Ciuleanu E, Bobos M, Kalogera-Fountzila A, Eleftheraki AG, Karayannopoulou G, et al. Induction chemotherapy followed by concomitant radiotherapy and weekly cisplatin versus the same concomitant chemoradiotherapy in patients with nasopharyngeal carcinoma: a randomized phase II study conducted by the Hellenic Cooperative Oncology Group (HeCOG) with biomarker evaluation. Ann Oncol. 2012; 23: 427-35.

22. Peerawong T, Phungrassami T, Pruegsanusak K, Sangthong R. Comparison of Treatment Compliance and Nutritional Outcomes among Patients with Nasopharyngeal Carcinoma with and without Percutaneous Endoscopic Gastrostomy during Chemoradiation. Asian Pacific Journal of Cancer Prevention. 2012; 13: 5805-9.

23. Irungu CW, Oburra $\mathrm{HO}$, Ochola B. Prevalence and Predictors of Malnutrition in Nasopharyngeal Carcinoma. Clin Med Insights Ear Nose Throat. 2015; 8: 19-22. 